\title{
RHIC INJECTION KICKER POWER SUPPLY SYSTEM CONTROL, INTEGRATION AND COMMISSION*
}

\author{
W. Zhang, R. Sanders, W. Fisher, and L. Hoff \\ AGS Department, Brookhaven National Laboratory, Upton, NY 11973-5000
}

\begin{abstract}
The RHIC Yellow ring Fast Injection Kicker Power Supply System was successfully commissioned in January 1997 as part of the Sextant Test. Construction of an identical system for the Blue ring has been completed and will be commissioned during the 1999 RHIC start-up. Each of these Injection Kicker systems consists of four subsystems and a control system center. The four subsystems are located on a floating high voltage deck inside a protective caged room because of the unusual grounding of the structure. The kicker power supplies and auxiliary equipment jump to potentials as high as $10 \mathrm{kV}$ during each pulse and must be inaccessible during operation. The RHIC Injection Kicker Power Supply System Control, Integration and Commissioning results are presented in this paper.
\end{abstract}

\section{INTRODUCTION}

The RHIC injection kicker system modulators, including four Blumleins, auxiliary power supplies and controls, are secured inside a safety cage. The Blumleins are mounted on insulated stands and the high voltage power supplies, auxiliary power supplies and controls are located on an isolated floating deck. The deck equipment is powered through a set of six high voltage isolation transformers. The communication and control to the system are via fiber optic cables.

The design of the RHIC injection fast kicker power supply control architecture was driven by recent successful experience with the AGS G10 fast extraction kicker system. Also, concerns about maintaining a high level of kicker noise immunity for the computer control system made it necessary to use of optical fiber isolation for remote control to local control interfaces in both the AGS and Booster fast extraction kickers. The RHIC injection fast kicker power supply system also has the additional need to isolate the control system from the high voltage pulses present at the floating deck. The system is designed to allow full local control as well as remote computerised control functions.

The upper level remote controls are VME based systems. It includes an Allen-Bradley PLC 5/V40B for remote command and status readback interface, A V102 timing delay generator for power supply timing signals, and a VME waveform generator for power supply voltage reference and charging waveform readback.

\footnotetext{
* Work performed under the auspices of the U.S. Department of Energy
}

Two $50 \mathrm{kV}$ rated ALE 802L power supplies serve as charging supplies for each of two kicker systems, both having four modulators. The operating voltage is limited to $34 \mathrm{kV}$ by the power supply voltage limiting function, and by the interface reference voltage clamping circuit, to protect magnet. The voltage charging of the Blumlein pulse forming line(PFL), is limited to less than 20 millisecond to avoid the PFL arcing. A backup trigger is required to discharge the PFL during the beam gap, in the event of the occurrence of an extra charging cycle.

\section{SUBSYSTEM DESCRIPTION}

\subsection{Command Control and Status Monitoring}

The Allen-Bradley PLC based command control and status-monitoring system uses Data Highway Plus network architecture. It consists of a PanelView 1400E industrial control terminal and a VME based PLC for a remote control interface. Both are located in control racks outside the high voltage cage area. An Allen-Bradley PLC 5 series processor is mounted in a control rack on the high voltage floating deck. The PanelView terminal is used for local command control and status monitoring. The VME PLC processor and PanelView terminal communicate to the floating deck PLC processor through a pair of Allen-Bradley PLC Fiber-Optic converter modules. This PLC network has been fully tested and experienced no communication interruptions due to use of fiber-optic communication or network structure.

The PLC ladder logic program is based on an AGS standardized kicker power supply format. The VME PLC is shared with other RHIC injection line power supply equipment. It communicates to the injection line equipment through Remote $\mathrm{I} / \mathrm{O}$ network, and to the injection kicker system through $\mathrm{DH}+$ network.

\subsection{Charging Reference and Readback}

The high voltage power supply command reference signal is generated at the VME waveform generator. Its complementary unit "16-bit serial data link" performs analog to digital and digital to analog conversions. It communicates to kicker power supply interface unit with analog signals.

The fiber optic power supply interface pair I \& II, as shown in Figure 1, were designed to interface with ALE 402 and 802 series power supplies. Their function is to 
prevent the kicker and beam noise that might possibly be carried back from accelerator ring by high voltage cables, from interfering with other systems, especially the remote control equipment. The analog reference signal and charging waveform signal are converted by voltage to frequency converters that communicate through a fiber optic transmitter/receiver pair. They are converted back to analog signals by frequency to voltage converter units. The charging command signal is determined by "Start Charge" and "Stop Charge" triggers which, in turn, enable and inhibit the high voltage power supply output circuit. Its complementary bit controls a trigger delay generator and prohibits it from pulsing during high voltage power supply charging cycle. This reduces the chance of high voltage power supply converter damage and thyratron lock up. A local reference control function is included in both ends of the interface pair to allow local testing which is independent of the remote control network.

\subsection{Timing and Trigger}

The RF synchronised discharge trigger is distributed to the AGS extraction fast kickers and the RHIC injection fast kicker system via fiber links. Two DG535 pulse delay generators are used in each kicker system. An RF synchronised trigger initiates the first delay generator, which, in turn, triggers the second unit. The high current output pulses of the first delay generator drives a solid state high voltage pulse generator that drives the grid 1 of the CX1168-C thyratron. A second delay generator output pulse drives four "Hard Tube Trigger Generators", which deliver $5 \mathrm{kV}$ trigger pulses to the bias grid of the thyratron.

It was found during the 1997 Sextant test, that the commercial XD-7430/RD-7430 fiber optic transmitter/ receiver pair was affected by local noise. It will be replaced by transmitter/receiver circuits designed at the AGS. Also, during the Sextant Test, the RF trigger was linked through the fiber optic transmitter/receiver channel of the backup trigger of the power supply interface units. The output pulse time jitter of the solid-state trigger generator and the hard tube trigger generator are within sub-nanosecond range similar to the scope rating. We control the fine delay adjustment of the delay generators via its GPIB interface.

\subsection{Signal Monitoring}

The terminating resistor current and thyratron current are monitored by fast four-channel digital scopes. Their waveform data will be retrieved via GPIB upon request.

\subsection{Grounding Structure and Other Issues}

The grounding structure of the RHIC injection kicker system is different from AGS kicker systems. It uses a separate ground in the RHIC ring. The Blumlein PFL outer conductors are joined with the floating deck and associated equipment racks electrically. During pulsing they rise as high as $10 \mathrm{kV}$. This configuration has introduced noise spikes through the local ground. This noise is especially noticeable when the four PFLs are pulsed in a non-synchronised .

The kicker magnets are driven by two $50 \mathrm{ohm}$ lines in parallel and have a resistive termination. The load resistor value was reduced from $25 \mathrm{ohm}$ to $20 \mathrm{ohm}$ in order to mismatch the load thus lowering the magnet voltage and raising the current, [4, 5]. In this case, the reversed voltage and current imposed to thyratron might have to be considered carefully because that CX-1168C is not rated for reversed conduction. This reversing voltage and current might cause thyratron cathode arcing at high voltage, and affect auxiliary circuits. During the RHIC Sextant Test, all thyratron and electronic equipment had survived the PFL arcing, reverse voltage, grounding loop noise, etc. We will have to learn from future operations the reliability and survivability of the thyratrons as well as other equipment.

\section{CONCLUSIONS}

The RHIC injection kicker systems used as many AGS standard designs as possible. This was done to expedite construction, assembly and installation, as well as reduce the number of stocked operational spares.

The RHIC yellow ring fast injection kicker system was brought into operation in a few hours after completion of the system installation and check out. The performance of its kickers and controls was satisfactory as indicated in papers [1], [2], [3] and [6].

During the RHIC Dry Run, we tested the Yellow ring injection kicker system with local and remote control functions. The Blue ring injection kicker system has just been powered and is being checked out at this stage.

\section{REFERENCES}

[1] S. Peggs, "RHIC Status", Proceeding IEEE 1997 Particle Accelerator Conference, pp. 56-60.

[2] J. Wei, et. Al., "RHIC Sextant Test - Physics and Performance", Proceeding IEEE 1997 Particle Accelerator Conference, pp. 6971.

[3] F. Pilat, et. Al., "RHIC Sextant Test: Accelerator Systems and Performance", Proceeding IEEE 1997 Particle Accelerator Conference, pp. 98-100.

[4] H. Hahn and A. Ratti, "Equivalent circuit Analysis of the RHIC Injection Kicker", Proceeding IEEE 1997 Particle Accelerator Conference, pp. 216-218.

[5] H. Hahn and A. Ratti, "The Coupling Impedance of the RHIC Injection Kicker System", Proceeding IEEE 1997 Particle Accelerator Conference, pp. 1706-1708.

[6] H. Hahn and A. Ratti, "The RHIC Injection Kicker", Proceeding IEEE 1997 Particle Accelerator Conference, pp. 213-215. 


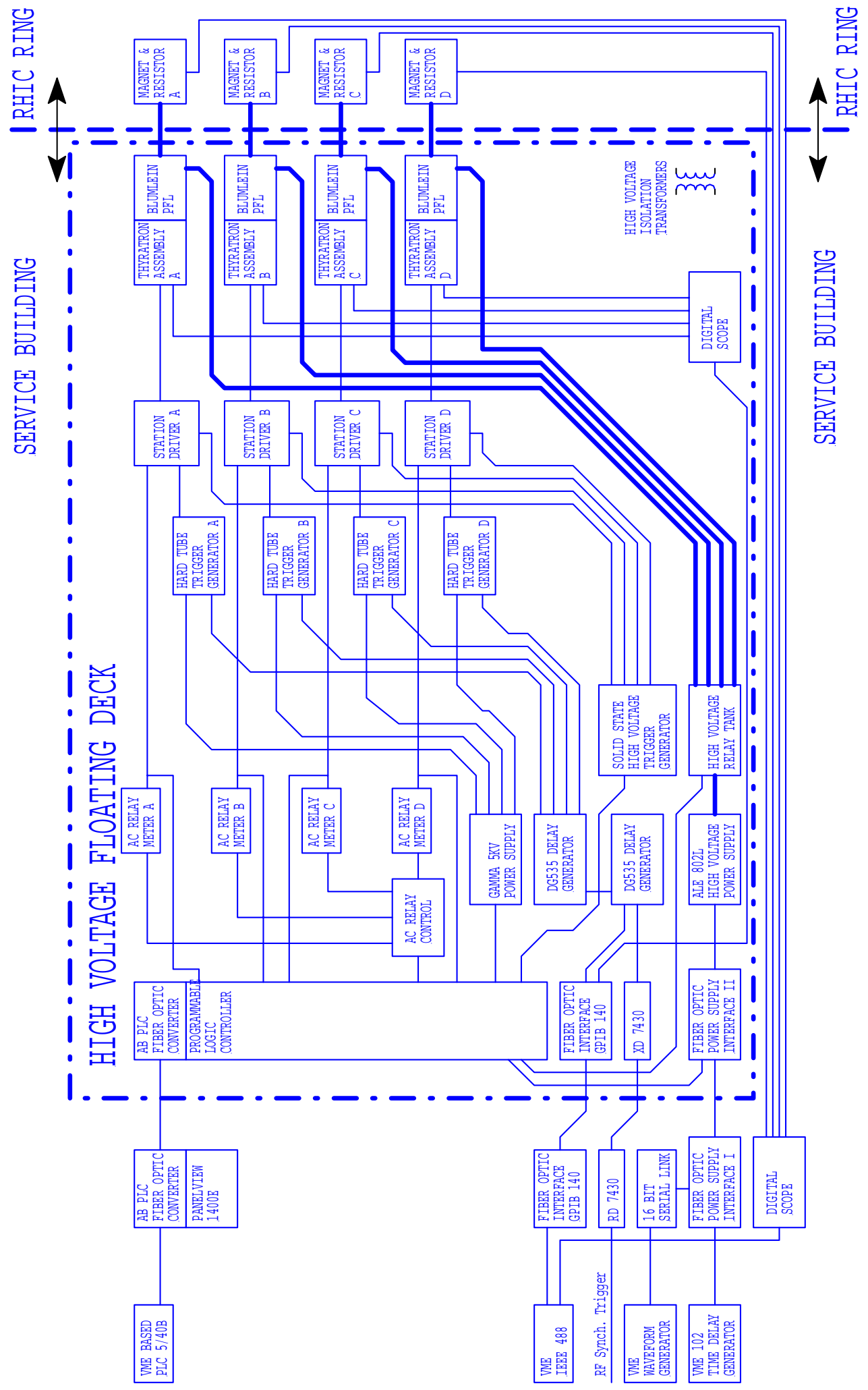

Figure 1. The simplified RHIC injection fast kicker control and auxiliary system block diagram. 